\title{
Body Mass Index Influences the Prognostic Impact of Combined Nuclear Insulin Receptor and Estrogen Receptor Expression in Primary Breast Cancer
}

\author{
Sofie Björner' ${ }^{1}$, Ann H. Rosendahl' ${ }^{1}$, Maria Simonsson ${ }^{1}$, Andrea Markkula', Karin Jirström ${ }^{1}$ \\ Signe Borgquist ${ }^{1,2}$, Carsten Rose ${ }^{3}$, Christian Ingvar ${ }^{4}$ and Helena Jernström ${ }^{1 *}$ \\ ${ }^{1}$ Faculty of Medicine, Department of Clinical Sciences Lund, Oncology and Pathology, Lund University, Lund, Sweden, \\ ${ }^{2}$ Clinical Trial Unit, Forum South, Skåne University Hospital, Lund, Sweden, ${ }^{3}$ CREATE Health and Department of \\ Immunotechnology, Lund University, Lund, Sweden, ${ }^{4}$ Department of Clinical Sciences Lund, Surgery, Lund University, \\ Skåne University Hospital, Lund, Sweden
}

OPEN ACCESS

Edited by:

Rosamaria Lappano, University of Calabria, Italy

Reviewed by: Maria Marino,

Università degli Studi Roma Tre, Italy

Luca De Toni,

Università degli Studi di Padova, Italy

*Correspondence:

Helena Jernström helena.jernstrom@med.Iu.se

Specialty section: This article was submitted to Cancer Endocrinology,

a section of the journal

Frontiers in Endocrinology

Received: 12 September 2017 Accepted: 09 November 2017 Published: 28 November 2017

Citation:

Björner S, Rosendahl AH,

Simonsson M, Markkula A, Jirström K, Borgquist S, Rose C, Ingvar $C$ and Jernström H (2017) Body Mass Index Influences the Prognostic Impact of Combined

Nuclear Insulin Receptor and Estrogen Receptor Expression in Primary Breast Cancer.

Front. Endocrinol. 8:332. doi: 10.3389/fendo.2017.00332
The prognostic importance of tumor-specific nuclear insulin receptor (InsR) expression in breast cancer is unclear, while membrane and cytoplasmic localization of InsR is better characterized. The insulin signaling network is influenced by obesity and may interact with the estrogen receptor $\alpha(E R \alpha)$ signaling. The purpose was to investigate the interplay between nuclear InsR, ER, body mass index (BMI), and prognosis. Tumorspecific expression of nuclear InsR was evaluated by immunohistochemistry in tissue microarrays from 900 patients with primary invasive breast cancer without preoperative treatment, included in a population-based cohort in Sweden (2002-2012) in relation to prognosis. Patients were followed for up to 11 years during which 107 recurrences were observed. Nuclear InsR ${ }^{+}$expression was present in 214 patients $(23.8 \%)$ and increased with longer time between surgery and staining $(P<0.001)$. There were significant effect modifications by ER status and BMI in relation to clinical outcomes. Nuclear InsR ${ }^{+}$conferred higher recurrence-risk in patients with $\mathrm{ER}^{+}$tumors, but lower risk in patients with $\mathrm{ER}^{-}$tumors $\left(P_{\text {interaction }}=0.003\right.$ ). Normal-weight patients with nuclear InsR $\mathrm{R}^{+}$tumors had higher recurrence-risk, while overweight or obese patients had half the recurrence-risk compared to patients with nuclear InsR- tumors ( $\left.P_{\text {interaction }}=0.007\right)$. Normal-weight patients with a nuclear InsR-/ER+ tumor had the lowest risk for recurrence compared to all other nuclear InsR/ER combinations $\left[\mathrm{HR}_{\mathrm{adj}} 0.50,95 \%\right.$ confidence interval $(\mathrm{Cl})$ : 0.25-0.97], while overweight or obese patients with nuclear InsR-/ER- tumors had the worst prognosis ( $\mathrm{HR}_{\text {adj }} 7.75,95 \% \mathrm{Cl}$ : 2.04-29.48). Nuclear InsR was more prognostic than ER among chemotherapy-treated patients. In summary, nuclear InsR may have prognostic impact among normal-weight patients with $\mathrm{ER}^{+}$tumors and in overweight or obese patients with ER- tumors. Normal-weight patients with nuclear InsR-/ER+ tumors may benefit from less treatment than normal-weight patients with other nuclear InsR/ER combinations. Overweight or obese patients with nuclear InsR ${ }^{-} / \mathrm{ER}^{-}$tumors may benefit from more tailored treatment or weight management.

Keywords: nuclear insulin receptor, estrogen receptor alpha, body mass index, breast cancer, prognosis, adjuvant breast cancer treatment 


\section{INTRODUCTION}

Overweight and obesity confer poor breast cancer prognosis such as increased risk for recurrence and death among patients with high body mass index (BMI) compared to lower BMI (1-4). There is an established connection between obesity and the insulin signaling pathway (5). One animal study indicated that the level of nuclear translocation of insulin receptor (InsR) may differ according to body weight (6). Whether the relationship between overweight/obesity and poor breast cancer prognosis is partly mediated through nuclear localization of InsR is unknown. The InsR is a tyrosine kinase receptor and belongs to the same receptor subfamily as the insulin-like growth factor 1 receptor (IGF1R). The two receptors share structural homology, signal via similar downstream signaling pathways, can form heterodimers, and both receptors are implicated in breast cancer $(7,8)$. The cytoplasmic and membrane tumor expression of InsR alone and in combination with IGF1R and phospho-IGF1R/InsR in relation to breast cancer outcome has previously been described in the same patient cohort as used in the present study. Patients with tumors expressing high abundance of IGF1R and InsR and concomitant activation were associated with the worst prognosis (9). In addition, a significant association between longer time between surgery and staining and weaker cytoplasmic and membrane InsR and phospho-IGF1R/InsR staining was reported.

Most studies of InsR have focused on the cytoplasmic and membranous localization of InsR, and mRNA levels of InsR including the two splice variants InsR-A and InsR-B and their associations with breast cancer features and prognosis $(10,11)$. It is well known that InsR internalization and downstream signaling activation of PI3K and AKT is followed by either receptor recycling to the membrane or lysosomal degradation $(12,13)$. In addition, InsR can translocate to the nucleus. Although not fully understood, both InsR and IGF1R have genomic functions involving the mitogen-activated protein kinase signaling pathway and cellular functions such as proliferation and migration (14-17). The IGF1R and estrogen receptor (ER) signaling pathways are subject to feedback cross-talk and are closely linked $(18,19)$. In a cell study, nuclear InsR-expression was higher in ER $\alpha$-depleted breast cancer cells and was able to suppress the IGF1R promotor activity in vitro $(16,20)$. Others have shown that the expression of hormone receptors such as ER was linked to BMI $(21,22)$ and that higher BMI was linked to higher estradiol levels in breast tissue in patients with $\mathrm{ER}^{+}$but not $\mathrm{ER}^{-}$tumors (23). Estrogen synthesis occurs though aromatization of androgens to estrogens by CYP19A1 in the adipose tissue, both locally in the breast as well as peripherally (3). In women, ligand levels are influenced by BMI. A higher BMI was associated with higher levels of circulating IGF-1, proinsulin, insulin, and C-peptide $(24,25)$. Studies in adipocytes and hepatocytes indicated that the internalization of the InsR only occurred after ligand stimulation $(26,27)$. Further, obesity was associated with hyperinsulinemia and caused abnormalities in the insulin signaling pathway (5), which may impact the expression and localization of insulinrelated factors, such as InsR, in breast cancer. Host factors such as BMI are therefore of importance in studies of prognostic and treatment predictive markers in breast cancer. Several treatment strategies directed against the IGF-1 axis are in clinical trials, but have mostly been disappointing in the clinical setting (28). Better prognostic and treatment predictive biomarkers are warranted. Neither the association between nuclear localization of InsR and patient's BMI or tumor ER status, nor the prognostic importance of the nuclear InsR have been established among breast cancer patients. In this study, we hypothesized that the prognostic value of tumor-specific nuclear InsR expression may differ according to the patients' BMI, tumor ER status, as well as type of adjuvant treatment.

\section{MATERIALS AND METHODS}

\section{Patients}

The breast cancer specimens were obtained from primary breast cancer patients in an ongoing population-based cohort, BC Blood Study, consisting of 1,116 patients. The patients were included between October 2002 and June 2012 at the Skåne University Hospital, Lund, Sweden as previously described (29). The included patients were between 24 and 99 years old and had no previous history of cancer within the last ten years. A research nurse measured the patients' anthropometric factors: weight, height, waist and hip circumference, and breast volume $(30,31)$ prior to surgery. In addition, patients were asked to fill in questionnaires regarding lifestyle factors as well as reproductive history and use of exogenous hormones or other medications during the past week. Patients were classified as diabetic if they self-reported use of any kind of antidiabetic treatment.

Clinicopathological data and information about breast cancer recurrences and deaths were obtained from medical records, pathology reports, the Regional Tumor Registry and the Population Registry (32-34). Adjuvant breast cancer treatment was administered according to standard of care and was recorded until the first breast cancer event. In patients without any breast cancer events, treatments were recorded until last follow-up or death prior to July 1,2014 . Patients may have received more than one type of adjuvant treatment during follow-up. As of November 2005, human epidermal growth factor receptor 2 (HER2) evaluation was introduced and trastuzumab was included in the adjuvant treatment setting. These were entered as a covariate in subgroup analyses of patients. Aromatase inhibitors (AIs) are mainly offered to postmenopausal patients and the impact of nuclear InsR on AI-treatment response was therefore only analyzed among patients $\geq 50$ years.

This study was carried out in accordance with the recommendations of the local ethics committee at Lund University with written informed consent from all patients. All patients gave written informed consent in accordance with the Declaration of Helsinki. The protocol was approved by the local ethics committee at Lund University (Dnr 75-02, Dnr 37-08, Dnr 658-09, Dnr 58-12, Dnr 379-12, Dnr 227-13, Dnr 277-15, and Dnr 458-15).

The final study cohort consisted of 900 patients after excluding patients who received preoperative treatment $(n=51)$, patients with only ductal carcinoma in situ $(n=39)$, patients with distant 
metastasis $\leq 0.3$ years from baseline $(n=8)$, or patients with no evaluable invasive tumor tissue on tissue microarray (TMA) $(n=118)$. The report followed the Reporting recommendations for tumor MARKer prognostic studies (REMARK) criteria (35).

\section{TMA and Immunohistochemistry}

Representative tumor regions of formalin-fixed paraffinembedded tissue blocks were collected from surgical specimens and assembled in a TMA containing duplicate $1.0 \mathrm{~mm}$ cores, using a semi-automated tissue array device (Beeches instruments, Sun Prairie, WI, USA). Freshly cut sections were automatically deparaffinized and pretreated using the PT Link system (DAKO, Glostrup, Denmark). All slides were stained simultaneously in one batch to obtain identical conditions in order to minimize staining variation due to technical factors. Immunohistochemistry was performed using the Autostainer Plus from DAKO with the EnVision FLEX high-pH kit, according to the manufacturer's instructions (DAKO, Glostrup, Denmark) with the InsR ( $\beta$-subunit) (GR36, Calbiochem; dilution 1:50) antibody. The InsR antibody detects the $\beta$ subunit, which is identical for the isoforms InsR-A and InsR-B. InsR expression in the present study thus reflects the total amount of InsR. ER $\alpha$ was routinely stained for clinical purposes at the Department of pathology in Lund and $\mathrm{ER} \alpha$ status was obtained from pathology reports (32-34). In Sweden, an ER $\alpha$ cutoff of $>10 \%$ of stained nuclei is still used in the clinic compared to the $1 \%$ cutoff that is used in other countries.

The immunohistochemical staining was evaluated by two independent observers ( $\mathrm{SBj}, \mathrm{AR}$ ) blinded to tumor characteristics and patient information. Reexamination was performed in case of discrepancy (1.5\%) until consensus was reached. The evaluated tumors were divided into negative or any positive nuclear staining. In case of bilateral tumors, all scores were based on the same tumor when evaluating the combined nuclear, cytoplasmic and membrane InsR expression. The prognostic impact of the cytoplasmic and membrane InsR staining intensities have been reported previously (9).

\section{Statistical Methods}

All statistical analyses were calculated using he SPSS software versions 22 (IBM). $\chi^{2}$ tests were used for analyses between dichotomized expression levels in different cellular compartments. Logistic regression tests were used for analyses between expression levels and categorical variables, presenting both crude odds ratios (OR) and adjusted $\mathrm{OR}\left(\mathrm{OR}_{\mathrm{adj}}\right)$ for time between surgery and staining (TBSAS, years). Kaplan-Meier curves and LogRank tests were used for univariable survival analyses. Cox regression was used for multivariable analyses providing hazard ratios (HRs) with 95\% confidence intervals (CIs) adjusted for age (continuous), invasive tumor size ( $\geq 21 \mathrm{~mm}$ or skin or muscular involvement), any axillary lymph node involvement, histological grade III, ER status, BMI ( $\left.\geq 25 \mathrm{~kg} / \mathrm{m}^{2}\right)$, TBSAS (years), and treatments. Two-way interaction terms between nuclear InsR and BMI $\left(\geq 25 \mathrm{~kg} / \mathrm{m}^{2}\right)$, and between nuclear InsR and ER status, and three-way interaction terms between nuclear InsR, BMI ( $\geq 25 \mathrm{~kg} /$ $\mathrm{m}^{2}$ ), and ER status were calculated and used in adjusted Cox regression analyses to investigate potential effect modifications.
In case of bilateral tumors $(n=15)$, the highest score was applied. The tumor characteristics from the same tumor were used in all analyses where tumor characteristics were included. Sensitivity analyses were performed using the scores for the contralateral invasive tumor. Restriction analyses were performed where patients who reported pre-operative treatment with anti-diabetic medications were excluded.

Breast cancer events were defined as local or regional recurrences, contralateral cancer or distant metastasis, and the time to event is referred to as event-free survival (EFS). For distant metastasis-free survival (DMFS), only distant metastases were considered events, and death due to any cause was the only considered event for overall survival (OS). Patients without events were censored at the last follow-up or death prior to July 1, 2014. Patients were followed from inclusion to the first breast cancer event, distant metastasis, or death, respectively.

Power calculations assuming 900 patients with an accrual interval of 10 years and additional follow-up time of two years and a frequency of $25 \%$ of positive nuclear InsR tumors showed that the study was able to detect true HRs between 0.762 and 1.342 with $80 \%$ power and an $\alpha$ of $5 \%$ (36). All $P$-values presented are two-tailed and nominal and were not adjusted for multiple testing since this is an exploratory study. A $P$-value of less than 0.05 was considered significant.

\section{RESULTS}

\section{Correlations between Localization of InsR in Different Cellular Compartments and Year of Surgery}

Figure 1A illustrates the distribution of nuclear InsR staining. There were 900 tumors (88.4\%) with available nuclear InsR scores of which 214 (23.8\%) tumors were positive for nuclear InsR. There was a significant association between longer TBSAS and positive nuclear staining: nuclear $\operatorname{InsR}^{+}$: 9.0 years (IQR 7.0-10.0, $P<0.001$ ), nuclear InsR ${ }^{-}$: 5.0 years (IQR 3.0-8.0), indicating that the proportion of patients with nuclear InsR ${ }^{+}$ tumors decreased over time with the highest proportion observed among patients included before 2006 (Figure 1B). There were significant inverse correlations in all time intervals between nuclear expression of InsR and membrane expression (negative, positive; all $r_{\mathrm{s}} \leq-0.15$, all $P$-values $\left.\leq 0.047\right)$. For cytoplasmic intensity (negative, weak, moderate, strong) and dichotomized cytoplasmic InsR expression (negative/weak versus moderate/ strong) there were inverse correlations that were significant in all time intervals with the exception of the years 2008-2009 (all $r_{\mathrm{s}} \leq-0.12$, all $P$-values $\left.\leq 0.048\right)$. InsR was rarely present in all three compartments simultaneously.

\section{Nuclear InsR in Relation to Tumor and Patient Characteristics}

Tables 1 and 2 present both crude and TBSAS adjusted associations between patient and tumor characteristics and nuclear InsR expression. In the crude analyses in Table 1, nuclear InsR ${ }^{+}$ was significantly associated with lower weight, BMI, and waistto-hip ratio, as well as younger age. These factors were no longer 
significant after adjustment for TBSAS. In both the crude and the adjusted models in Table 2, nuclear InsR ${ }^{+}$was significantly positively associated with HER2 amplification and postoperative adjuvant trastuzumab treatment, but not with $\mathrm{ER}^{+}$status. Nuclear InsR ${ }^{+}$was also significantly positively associated with postoperative adjuvant chemotherapy in the adjusted model.

\section{Nuclear InsR Expression in Relation to BMI and Year of Surgery}

There was an inverse correlation between nuclear InsR expression and TBSAS in all three patients groups stratified by BMI status as normal-weight, overweight, and obese $(<25.00,25.00-25.99$, 30.00+; all $r_{\mathrm{s}} \leq-0.31$, all $P$-values $\left.<0.001\right)$. In contrast, there

A

Patients with primary breast cancer
Oct 2002-June 2012
$n=1,116$

Preoperative treatment

$n=51$

In situ carcinoma

$n=39$

Distant metastasis $\leq 0.3$

years from inclusion

$n=8$

Invasive breast cancer

$n=1,018$

Missing on TMA or

missing InsR score

$n=118$

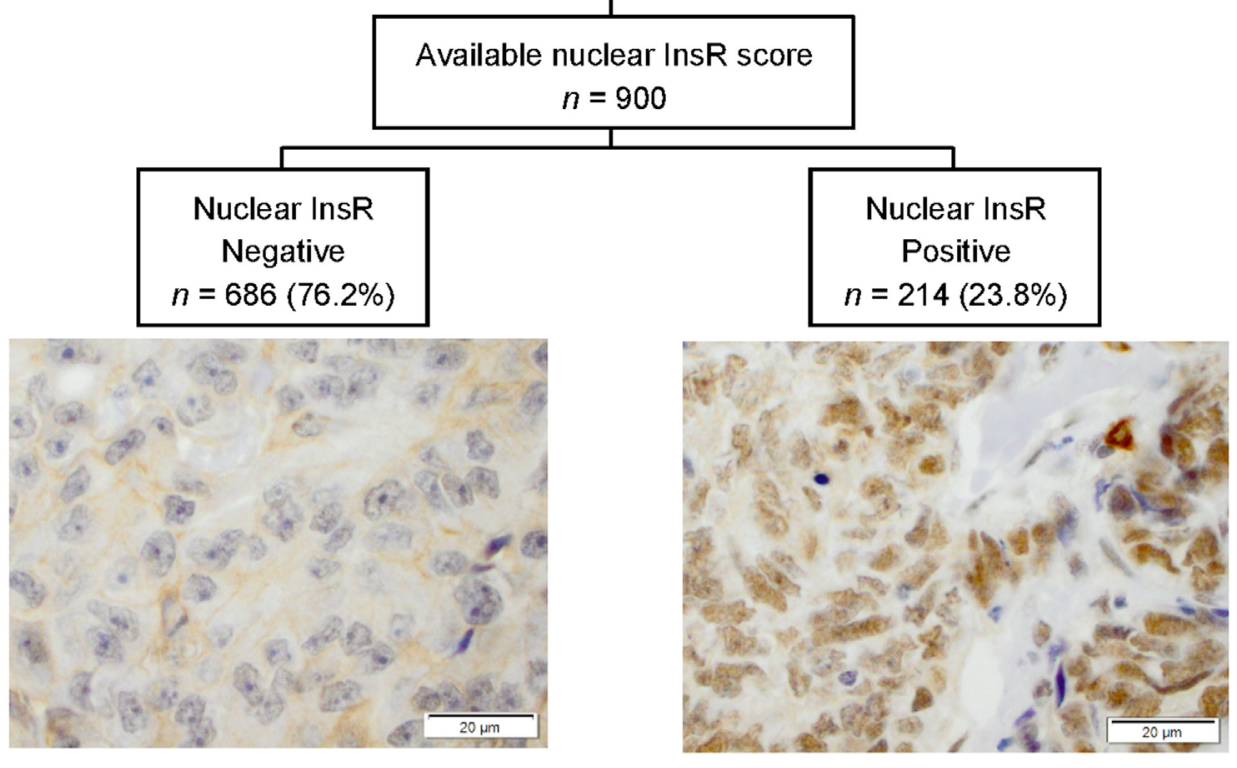




\section{B}

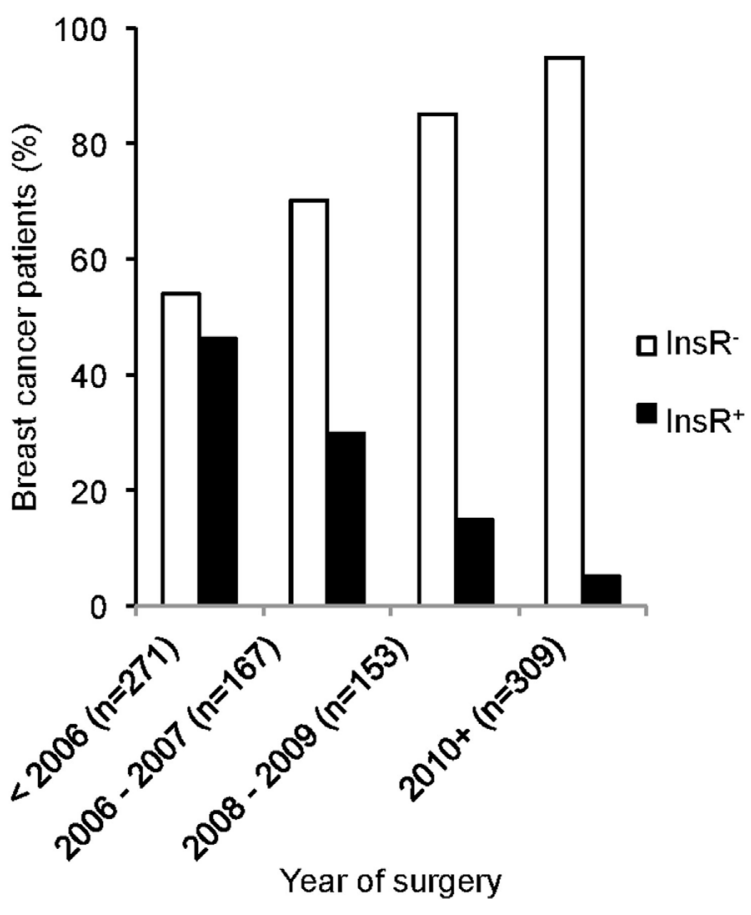

FIGURE 1 | (A) Flow chart of study population and marker distribution with representative images of negative and positive nuclear insulin receptor (InsR) expressions, scale bar $=20 \mu \mathrm{m}$. (B) Nuclear InsR expression in relation to year of surgery.

were no associations between nuclear InsR and BMI in the different time intervals (<2006, 2006-2007, 2008-2009, 2010+), indicating that the association between nuclear InsR and BMI did not change over time.

\section{Nuclear InsR As a Prognostic Marker Alone and in Relation to BMI}

Patients were followed for up to 11 years with a median follow-up of 5.0 years for patients still at risk. Of the 900 patients, 107 had had any breast cancer event and 67 of these patients had a distant metastasis. Eighty-six patients died due to any cause and 52 of these patients had had a reported breast cancer event prior to death.

Nuclear InsR was not a prognostic marker for EFS, DMFS, or OS among all patients. However, there were significant effect modifications depending on BMI on the associations between nuclear InsR and EFS $\left(P_{\text {interaction }}=0.007\right)$, DMFS $\left(P_{\text {interaction }}=0.040\right)$, and OS $\left(P_{\text {interaction }}=0.027\right)$. After adjustment for prognostic factors and TBSAS, normal-weight patients with nuclear InsR ${ }^{+}$tumors had a non-significant increased risk for any breast cancer event and non-significant shorter DMFS and OS. In contrast, overweight or obese patients with nuclear InsR ${ }^{+}$ tumors had a significantly decreased risk of any breast cancer event [adjusted $\mathrm{HR}\left(\mathrm{HR}_{\mathrm{adj}}\right)$ 0.48, 95\% CI: 0.25-0.92], and of death due to any cause $\left(\mathrm{HR}_{\mathrm{adj}} 0.35,95 \% \mathrm{CI}: 0.17-0.72\right)$, and a nonsignificant longer DMFS.

\section{Combined Nuclear InsR/ER As Prognostic Marker}

There were also significant effect modifications of ER status on the association between nuclear InsR and EFS where nuclear InsR ${ }^{+}$ expression in $\mathrm{ER}^{+}$tumors was associated with a non-significant higher risk of any breast cancer event and with lower risk in patients with $\mathrm{ER}^{-}$tumors $\left(P_{\text {interaction }}=0.003\right)$. A similar effect modification between nuclear InsR and ER status was observed regarding DMFS $\left(P_{\text {interaction }}=0.043\right)$, but not with respect to OS. A combined nuclear InsR/ER expression score for each tumor was therefore calculated resulting in four different groups: $\mathrm{InsR}^{-} / \mathrm{ER}^{+}$ $(n=609), \mathrm{InsR}^{+} / \mathrm{ER}^{+}(n=182), \mathrm{InsR}^{+} / \mathrm{ER}^{-}(n=32)$, and InsR ${ }^{-}$ $\mathrm{ER}^{-}(n=76)$. One patient had missing ER status.

\section{Prognostic Impact of Nuclear InsR/ER Expression}

The independent prognostic impact of nuclear InsR alone and in combination with ER and BMI on EFS is presented in different multivariable models in Supplementary Table 1. Overall, among all patients before and after BMI stratification, ER status seemed to impact prognosis more than nuclear InsR status (Figure 2), except among normal-weight patients regarding EFS (Figure 2B). However, irrespective of BMI, patients with nuclear InsR ${ }^{-} / \mathrm{ER}^{-}$tumors had, compared to all other nuclear InsR/ER combinations, the worst EFS ( $\mathrm{HR}_{\text {adj }} 5.03$, 95\% CI: 1.74-14.54; Figure 2A) and DMFS ( $\mathrm{HR}_{\mathrm{adj}}$ 3.57, 95\% CI: 1.04-12.23; Figure 2D), but not OS (Figure 2G). 


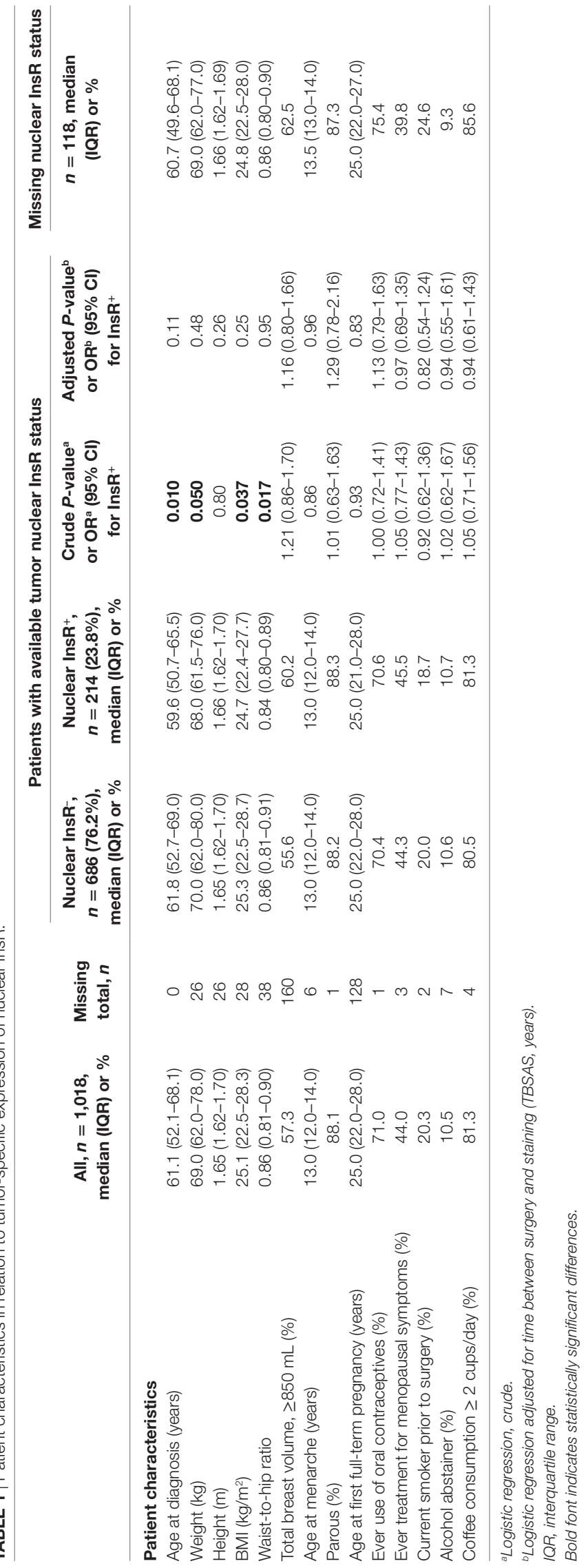

\section{Prognostic Impact of Nuclear InsR/ER in Relation to BMI}

After stratification by BMI, the increased risk was mainly limited to the overweight or obese group of patients with nuclear InsR ${ }^{-} /$ $\mathrm{ER}^{-}$tumors compared to all other nuclear InsR/ER combinations $\left(\mathrm{HR}_{\text {adj }} 7.75,95 \%\right.$ CI: 2.04-29.48; Figure 2C). Overweight or obese patients with a nuclear InsR ${ }^{-} /$ER $^{-}$tumors had also highest risk of DMFS (HR $\mathrm{Hdj}_{\text {adj }} 3.90,95 \%$ CI: 0.97-15.74; Figure 2F) and OS (HR $\mathrm{Hdj}_{\mathrm{adj}}$ 2.72, 95\% CI: 0.79-9.35; Figure 2I) compared to all other nuclear InsR/ER combinations. Conversely, overweight or obese patients with nuclear InsR ${ }^{+} / \mathrm{ER}^{+}$tumors had a significantly lower risk for death $\left(\mathrm{HR}_{\text {adj }} 0.39,95 \% \mathrm{CI}\right.$ : 0.17-0.89) compared to all other nuclear InsR/ER combinations. Normal-weight patients with a nuclear InsR $/ \mathrm{ER}^{+}$tumor, which constituted two thirds of the patients, had the lowest risk for recurrence compared to all other nuclear InsR/ER combinations $\left(\mathrm{HR}_{\mathrm{adj}} 0.50,95 \%\right.$ CI: 0.25-0.97; Figure 2B). There was no significant three-way interaction between nuclear InsR, ER, and BMI with respect to EFS when a formal three-way interaction analysis was performed.

\section{The Prognostic Impact of Combined Nuclear InsR/ER or Individual Nuclear InsR in Relation to Adjuvant Treatment and BMI Status}

The combined expression of nuclear InsR and ER was investigated in relation to prognosis in different breast cancer treatment groups. For endocrine treatment, only patients with $\mathrm{ER}^{+}$tumors were included. Any breast cancer event was used as a marker for poor adjuvant treatment response.

\section{Chemotherapy}

There were significant effect modifications of BMI on the association between nuclear InsR and EFS $\left(P_{\text {interaction }}=0.003\right)$, and DMFS $\left(P_{\text {interaction }}=0.047\right)$, but not for OS. Overweight or obese chemotherapy-treated patients with nuclear $\mathrm{InsR}^{+}$-expressing tumors had a significantly lower risk of any event $\left(\mathrm{HR}_{\mathrm{adj}} 0.12\right.$, 95\% CI: 0.02-0.69), while opposite results were observed among chemotherapy-treated normal-weight patients with nuclear InsR ${ }^{+}$-expressing tumors $\left(\mathrm{HR}_{\text {adj }} 2.61,95 \% \mathrm{CI}\right.$ : 0.76-9.00). In all chemotherapy-treated patients, combined nuclear InsR/ER was not a prognostic marker for EFS (Figure 3A), DMFS, or OS. The lowest risk for recurrence was again observed among normalweight patients with nuclear InsR $\mathrm{R}^{-} / \mathrm{ER}^{+}$tumors compared to all other nuclear InsR/ER combinations ( $\mathrm{HR}_{\mathrm{adj}} 0.08,95 \% \mathrm{CI}$ : 0.01-0.52; Figure 3B), while overweight or obese patients with nuclear InsR $/ \mathrm{ER}^{-}$tumors had the worst prognosis compared to all other nuclear InsR/ER combinations ( $\mathrm{HR}_{\text {adj }} 6.66,95 \% \mathrm{CI}$ : 1.14-38.94; Figure 3C). In conclusion, nuclear InsR conferred more prognostic information than ER status in chemotherapytreated patients after stratification by BMI. Overweight or obese patients with nuclear InsR $\mathrm{R}^{-} / \mathrm{ER}^{-}$tumors had the worst prognosis compared to other groups.

\section{Radiotherapy}

In radiotherapy-treated patients overall, combined nuclear InsR/ ER was a prognostic marker for EFS (Figure 3D), DMFS and OS, 
TABLE 2 | Tumor characteristics and treatments in relation to tumor-specific expression of nuclear InsR.

\begin{tabular}{|c|c|c|c|c|c|c|c|}
\hline & \multirow[b]{2}{*}{$\begin{array}{c}\text { All, } \\
N=1,018, \\
n(\%)\end{array}$} & \multirow[b]{2}{*}{$\begin{array}{c}\text { Missing } \\
\text { total, } \\
n\end{array}$} & \multicolumn{4}{|c|}{ Patients with available tumor nuclear InsR status } & \multirow{2}{*}{$\begin{array}{c}\text { Missing nuclear } \\
\text { InsR status } \\
\begin{array}{c}n=118 \\
n\end{array}\end{array}$} \\
\hline & & & $\begin{array}{c}\text { Nuclear InsR-, } \\
n=686(76.2 \%) \\
n(\%)\end{array}$ & $\begin{array}{c}\text { Nuclear InsR } \\
n=214(23.8 \%) \\
n(\%)\end{array}$ & $\begin{array}{c}\text { Crude OR } \\
(95 \% \mathrm{Cl}) \text { for InsR }\end{array}$ & $\begin{array}{c}\text { Adjusted OR } \\
\text { (95\% Cl) for InsR }\end{array}$ & \\
\hline \multicolumn{8}{|l|}{ Tumor characteristics } \\
\hline Invasive tumor size & & 0 & & & & & \\
\hline$\leq 20 \mathrm{~mm}$ & $741(72.8)$ & & $498(72.6)$ & $154(72.0)$ & Ref. & Ref. & 89 \\
\hline $\begin{array}{l}\geq 21 \mathrm{~mm} \text { and skin or muscular } \\
\text { involvement independent of size }\end{array}$ & $277(27.2)$ & & $188(27.4)$ & $60(28.0)$ & $1.03(0.73-1.45)$ & $1.17(0.81-1.70)$ & 29 \\
\hline Axillary lymph node involvement & & 2 & & & & & \\
\hline 0 & $627(61.7)$ & & $426(62.3)$ & $121(56.5)$ & Ref. & Ref. & 80 \\
\hline $1-3$ & $303(29.8)$ & & $201(29.4)$ & $72(33.6)]$ & $1.27(0.93-1.73)$ & $1.30(0.93-1.82)$ & 30 \\
\hline$\geq 4$ & $86(8.5)$ & & $57(8.3)$ & $21(9.8)]$ & & & 8 \\
\hline Histologic grade & & 1 & & & & & \\
\hline I & $255(25.1)$ & & $153(22.3)$ & $65(30.4)]$ & Ref. & Ref. & 37 \\
\hline$\|$ & $505(49.7)$ & & $351(51.2)$ & $100(46.7]$ & & & 54 \\
\hline III & 257 (25.3) & & $182(26.5)$ & $49(22.9)$ & $0.82(0.57-1.18)$ & $1.11(0.75-1.64)$ & 26 \\
\hline Histologic type & & 64 & & & & & \\
\hline Ductal & 766 (80.3) & & $518(80.4)$ & $170(85.4)$ & Ref. & Ref. & 78 \\
\hline Lobular & $111(11.6)$ & & $77(12.0)$ & $15(7.5)$ & $0.59(0.33-1.06)$ & $0.56(0.30-1.05)$ & $19^{c}$ \\
\hline Other/mixed & $77(8.1)$ & & $49(7.6)$ & $14(7.0)$ & $0.87(0.47-1.62)$ & $0.66(0.34-1.30)$ & $14^{\circ}$ \\
\hline \multicolumn{8}{|l|}{ Hormone receptor status } \\
\hline $\mathrm{ER}^{+}(>10 \%)$ & 894 (87.9) & 1 & 609 (88.9) & $182(85.0)$ & $0.71(0.46-1.11)$ & $0.69(0.42-1.12)$ & 103 \\
\hline $\mathrm{PR}^{+}(>10 \%)$ & $722(71.0)$ & 1 & $492(71.8)$ & $146(68.2)$ & $0.84(0.60-1.17)$ & $0.91(0.63-1.30)$ & 84 \\
\hline HER2 amplification ${ }^{d}$ & $83(12.2)$ & 49 & $46(9.0)$ & $18(19.6)$ & $2.45(1.35-4.46)$ & $2.82(1.48-5.37)$ & $19^{c}$ \\
\hline \multicolumn{8}{|l|}{ Treatment by last follow-up } \\
\hline Ever chemotherapy & $259(25.4)$ & 0 & $177(25.8)$ & $51(23.8)$ & $0.90(0.63-1.29)$ & $1.51(1.01-2.25)$ & 31 \\
\hline Ever radiotherapy & $641(63.0)$ & 0 & $439(64.0)$ & $135(63.1)$ & $0.96(0.70-1.32)$ & $1.02(0.72-1.43)$ & 67 \\
\hline Ever trastuzumab ${ }^{d}$ & $65(8.9)$ & 0 & $37(6.8)$ & $14(14.3)$ & $2.30(1.19-4.44)$ & $3.39(1.66-6.91)$ & $14^{c}$ \\
\hline \multicolumn{8}{|l|}{$\mathrm{ER}^{+}$only } \\
\hline Ever endocrine therapy & $695(77.7)$ & 0 & $480(78.8)$ & $143(78.6)$ & $0.99(0.66-1.48)$ & $0.95(0.61-1.47)$ & 72 \\
\hline Ever tamoxifen & $528(59.1)$ & 0 & $357(58.6)$ & $115(63.2)$ & $1.21(0.86-1.71)$ & $0.81(0.55-1.19)$ & 56 \\
\hline Ever aromatase inhibitor & $346(38.7)$ & 0 & $230(37.8)$ & $78(42.9)$ & $1.24(0.88-1.73)$ & $1.25(0.86-1.80)$ & 38 \\
\hline
\end{tabular}

a Logistic regression, crude.

${ }^{b}$ Logistic regression adjusted for time between surgery and staining (TBSAS, years).

'Significant difference between included and excluded patients.

${ }^{d} H E R 2$ was routinely analyzed in patients < 70 years with invasive tumors as of November $2005(n=732)$.

ePatients may have received more than one type of treatment prior to any event.

Bold font indicates statistically significant differences.

but this was driven by ER status for all three outcomes. There was a borderline interaction between BMI and nuclear InsR for EFS $\left(P_{\text {interaction }}=0.10\right)$, and significant interactions for both DMFS $\left(P_{\text {interaction }}=0.038\right)$ and OS $\left(P_{\text {interaction }}=0.042\right)$. In normal-weight patients, three out of six patients with nuclear InsR ${ }^{+} / \mathrm{ER}^{-}$tumors had an early event (Figure 3E), although not significant in the multivariable model. Similar to the overweight or obese chemotherapy-treated group, overweight or obese radiotherapytreated patients with nuclear InsR $\mathrm{R}^{-} / \mathrm{ER}^{-}$tumors had the highest risk for recurrence compared to all other nuclear InsR/ER combinations $\left(\mathrm{HR}_{\mathrm{adj}}\right.$ 12.62, 95\% CI: 1.51-105.27; Figure 3F). In summary, the combined prognostic impact of nuclear InsR/ER in radiotherapy-treated patients was predominantly due to the ER status. Overweight or obese patients with nuclear InsR ${ }^{-} / \mathrm{ER}^{-}$ tumors again had the worst prognosis compared to other groups.

\section{Endocrine Treatment}

Among tamoxifen (TAM)-treated patients with $\mathrm{ER}^{+}$tumors, nuclear InsR $\mathrm{R}^{+}$expression was weakly associated with shorter EFS
(Figure 3G), although not significant in the multivariable model. There was a borderline significant effect modification depending on BMI between nuclear InsR and EFS ( $\left.P_{\text {interaction }}=0.10\right)$. Multivariable survival analyses revealed that nuclear InsR only had prognostic impact among normal-weight TAM-treated patients. A significant increased risk of any breast cancer event was seen among normal-weight patients with nuclear InsR ${ }^{+}$ tumors $\left(\mathrm{HR}_{\mathrm{adj}}\right.$ 2.84, 95\% CI: 1.10-7.32; Figure $\left.3 \mathrm{H}\right)$ but not among overweight or obese patients (Figure 3I). In all TAMtreated patients with $\mathrm{ER}^{+}$tumors, nuclear InsR ${ }^{+}$tumors were associated with a 2 -fold increased risk for shorter DMFS $\left(\mathrm{HR}_{\mathrm{adj}}\right.$ 2.30, 95\% CI: 1.02-5.19). However, as observed for EFS, this increased risk was limited to normal-weight patients $\left(H_{\mathrm{adj}}\right.$ 3.70, 95\% CI: 1.01-13.60), while there was no increased risk in overweight or obese patients $\left(P_{\text {interaction }}=0.12\right)$. No significant associations were observed between nuclear InsR and OS in TAM-treated patients.

There was a borderline significant interaction between BMI and nuclear InsR on EFS $\left(P_{\text {interaction }}=0.069\right)$ but not 


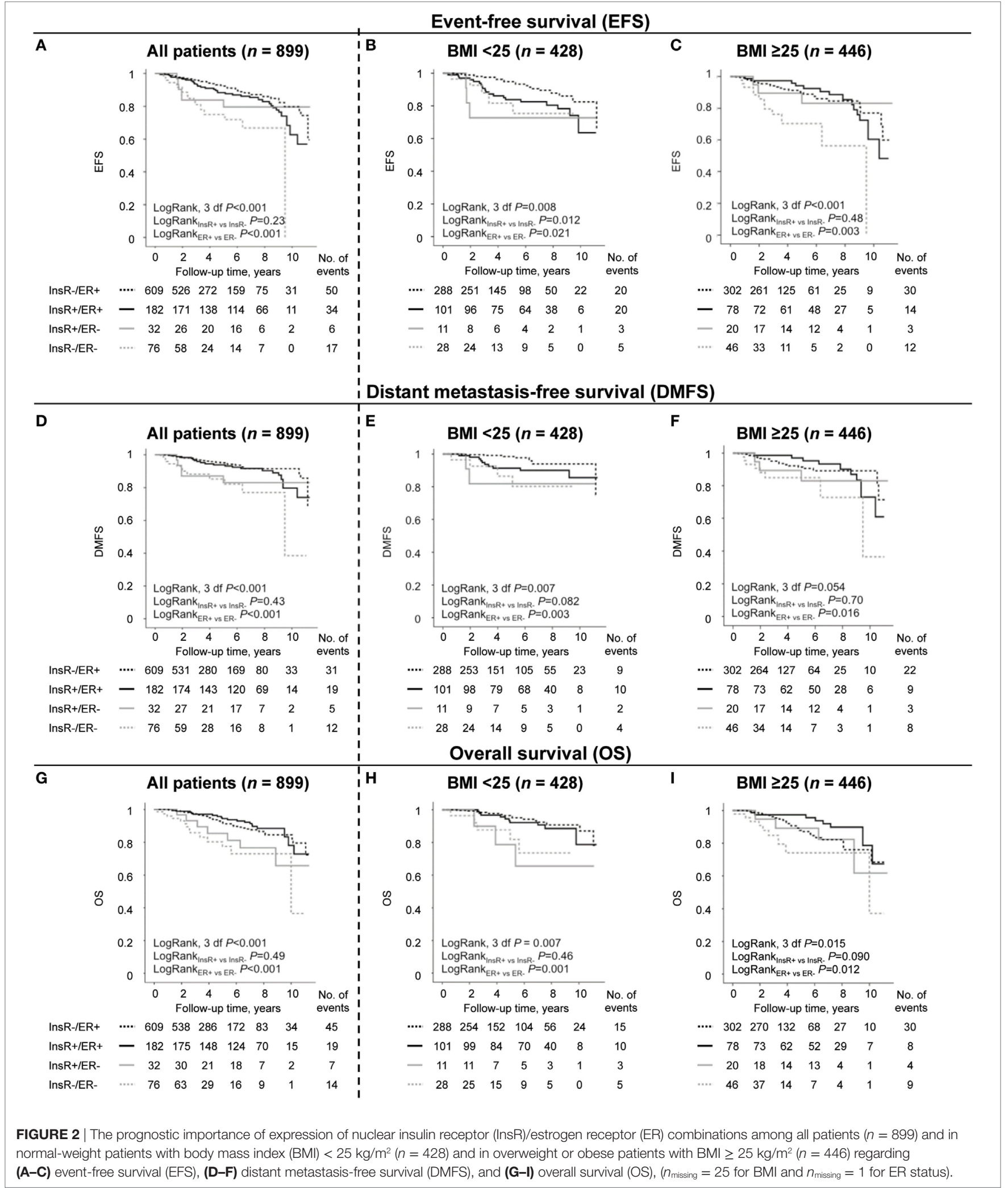

on DMFS or OS among AI-treated patients aged $\geq 50$ years. The effect estimates were similar to those seen in TAMtreated patients. However, nuclear InsR expression was not an independent prognostic marker for EFS, DMFS, or OS among AI-treated patients aged $\geq 50$ years, irrespective of BMI status. 


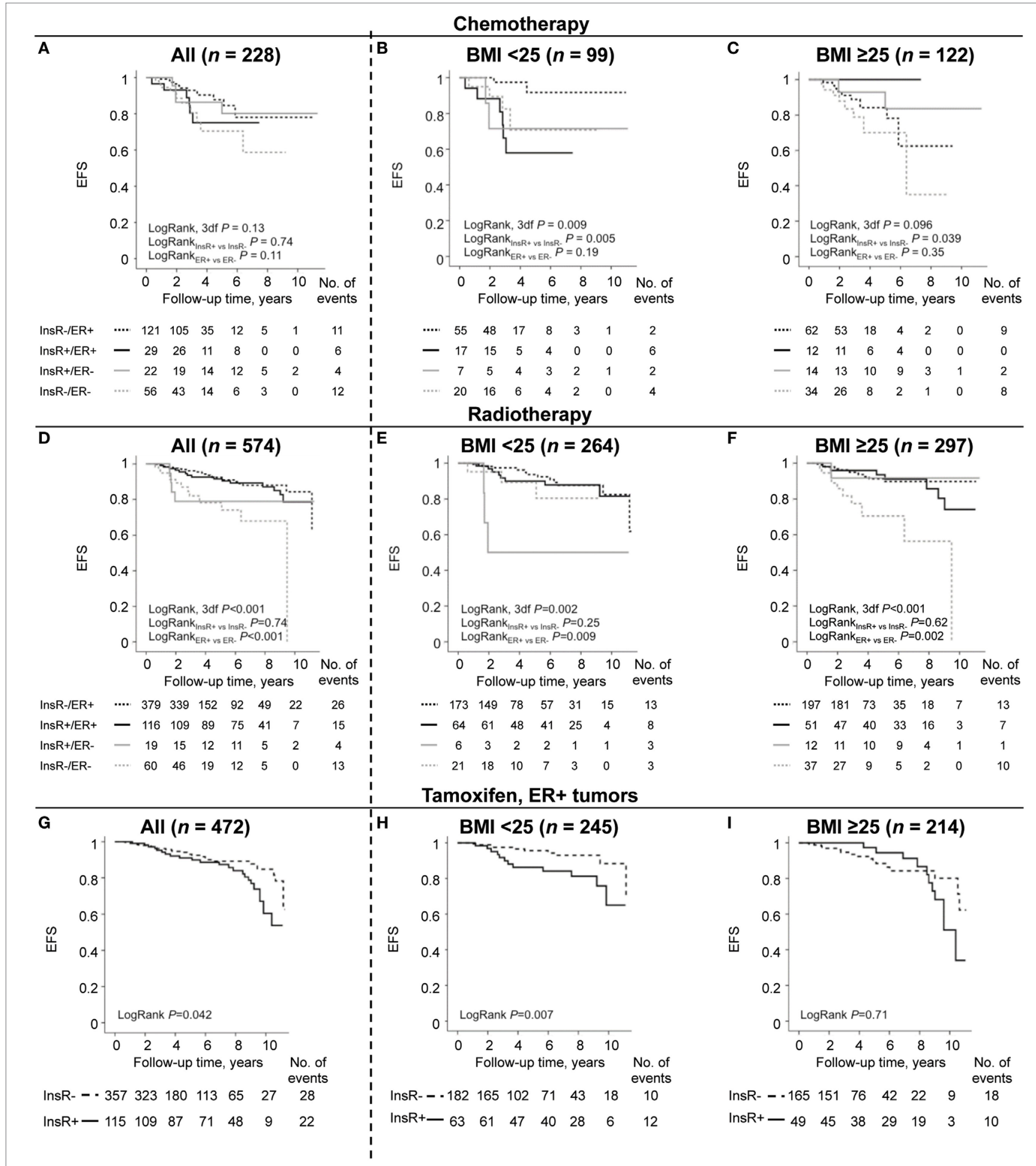

FIGURE 3 | Combinations of nuclear insulin receptor (InsR)/estrogen receptor (ER) as prognostic marker for event-free survival (EFS) for all patients and in patients stratified by body mass index (BMI). (A-C) Chemotherapy-treated patients, (D-F) radiotherapy-treated patients, and (G-I) tamoxifen (TAM)-treated patients with ER ${ }^{+}$ tumors.

In brief, in normal-weight TAM-treated patients with $\mathrm{ER}^{+}$ tumors, nuclear InsR may confer poor prognosis, but not in patients with higher BMI.

\section{Sensitivity and Restriction Analyses}

Sensitivity analyses were performed due to the 15 patients with available bilateral tumors. Nine patients had evaluable invasive 
tumor tissue on the contralateral side and five tumors changed dichotomized status for the patients with two evaluable tumors. The effect estimates remained essentially the same in all sensitivity analyses using the dichotomized status for the contralateral tumor. In total there were six analyses that went from significant to borderline significant and three analyses that went from borderline to significant.

There were $29 / 898$ patients (3.2\%) who self-reported any kind of antidiabetic treatment based on the preoperative questionnaire (two missing) who were excluded in the restriction analyses. Among these 29 patients, there were two breast cancer events, no distant metastasis, and nine deaths. The frequency of nuclear InsR was non-significantly lower in patients who reported antidiabetic treatment (13.8\%) compared with other patients $(24.2 \%$; $P=0.20)$. There were ten analyses that changed from significant to borderline significant and four analyses that changed from borderline to significant, but the effect estimates remained similar.

\section{DISCUSSION}

This study suggests that nuclear localization of InsR has differential prognostic roles depending on the patients' BMI and ER status in contrast to membrane and cytoplasmic localized InsR (9). These findings are important since obesity is a global health concern associated with aberrant insulin sensitivity and also impaired breast cancer outcomes. InsR can translocate to the nucleus and act as a transcription factor, but the role of nuclear expressed InsR in breast cancer prognosis has to our knowledge not been previously investigated. In the current study, BMI only modified the association between nuclear InsR and clinical outcome, but did not impact the association between membrane and cytoplasmic InsR expression and clinical outcome. In vitro findings suggest that nuclear localization of InsR is higher in ER-depleted cells than in $\mathrm{ER}^{+}$cells (16), but in the present study there was no association between tumor-specific nuclear InsR and ER status in contrast to membrane and cytoplasmic InsR expression (9). Further, only the nuclear InsR expression was associated with HER2 amplification in contrast to membrane and cytoplasmic InsR expression (9).

Insulin receptor is part of the IGF-signaling network and there is crosstalk between this network and other signaling pathways such as ER $(20,37,38)$. The current study suggests that nuclear InsR may be of prognostic importance among normal-weight patients with $\mathrm{ER}^{+}$tumors and among overweight or obese patients with $\mathrm{ER}^{-}$tumors. Potential explanations for the observed differences in prognostic impact of nuclear InsR according to BMI include changes in methylations patterns (39), ligand levels (5, 23-25, 40), and altered intracellular receptor trafficking and recycling rates depending on ligand levels (41), as well as differential nuclear InsR suppression of the IGF1R promotor according to tumor ER status (16). These potential mechanisms may in part explain the observed effect modifications of BMI and ER on the clinical impact of nuclear InsR and need to be elucidated in future studies. Age and menopausal status also impact BMI (4), but in this study there was no significant interaction between nuclear InsR and age $\geq 50$ years (data not shown). Patients were therefore not stratified based on age. Finally, in addition to ER status, breast cancer is a heterogeneous disease with multiple subtypes (42), which may modify the prognostic importance of nuclear InsR.

Insulin has the ability to enhance the cytotoxic effect of chemotherapy in vitro (43), which may explain the observed decreased recurrence-risk among overweight or obese chemotherapytreated patients with nuclear InsR ${ }^{+}$tumors in this study. The interplay between InsR, ER and IGF1R emphasizes the importance of combining the expression of nuclear InsR and ER and their impact as prognostic and treatment predictive biomarkers needs further elucidation. Targeting of all three receptors may be more efficient since dual inhibition of InsR and IGF1R with tyrosine kinase inhibitor OSI-906 in combination with ER downregulator fulvestrant more effectively suppress hormone-independent tumor growth than either drug alone (44). The current study indicates that BMI may also yield additional treatment predictive information with respect to drugs targeting the IGF1R/InsR and ER pathways. Since there is an ongoing obesity epidemic, nuclear InsR, which appears to play a differential role in overweight or obese compared to normal-weight patients, warrants further investigation in an independent study, preferably in randomized clinical trials of IGF-targeting treatments.

One of the findings in this study was that nuclear InsR expression was associated with time between surgery and staining. Changes of the expression of immunohistochemical markers over time should be considered in the statistical modeling when evaluating the prognostic impact of new markers. There was a significant association between earlier year of surgery and positive nuclear InsR staining. However, nuclear expression was inversely correlated with cytoplasmic and membrane InsR expression, indicating that the total InsR expression was stable over time, while the localization of InsR differed. The fact that the total InsR expression was stable over time may be considered as an internal control and validate the immunohistochemical staining. Additionally, all immunohistochemistry was performed simultaneously in one single batch in order to minimize technical variation. There may be several explanations for different InsR localizations such as differences in pre-analytical handling of the tumor tissue and differences in the standard of care for the patients, including different glucose concentrations in the perioperative intravenous drip or different anesthetic agents. Use of betamethasone to counteract postoperative nausea may impact blood glucose levels $(45,46)$, which could potentially impact on InsR nuclear translocation $(26,27)$, but lies outside the scope of this study. The differences in nuclear InsR expression in relation to year of surgery have been taken into account in all the regression analyses where TBSAS have been adjusted for. The results were driven by patients included 2002-2007. There were few patients with nuclear InsR ${ }^{+}$tumors 2008-2012 and the prognostic importance of this marker in today's setting needs to be elucidated. In addition to adjustments for TBSAS, restriction and sensitivity analyses were also performed. The results remained essentially the same after exclusion of patients treated with antidiabetic medications and in sensitivity analyses for the patients with bilateral tumor tissue on the TMA.

This is a population-based cohort with high follow-up rates $(29,47,48)$. The Swedish population have in general a lower BMI compared to the North American population (49), which may 
impact the generalizability of the results. The vast majority of the patients' body measurements were taken preoperatively by a research nurse, which minimizes the risk for bias. HER2 status was only available as of November 2005, which is a weakness. The ER $\alpha$ cutoff of $>10 \%$ is still used in the clinic in Sweden and is higher than in other countries. However, over $85 \%$ of the patients still had $\mathrm{ER}^{+}$tumors.

In summary, the results from this study support that the prognostic value of nuclear InsR expression depends on cellular localization and that the prognostic value of nuclear InsR is dependent on ER and BMI status. Furthermore, this study highlights the importance of investigating possible changes in staining intensity and encourages that future evaluation of immunohistochemical markers should take the time between surgery and staining into consideration. Our results substantiated that nuclear InsR expression had a higher prognostic value than ER among chemotherapy-treated patients once BMI was taken into account. Conversely, ER was a better prognostic marker of radiotherapy response than nuclear InsR. The study further suggests that nuclear InsR may be of prognostic importance among normal-weight patients with $\mathrm{ER}^{+}$tumors and in overweight or obese patients with $\mathrm{ER}^{-}$tumors. The normal-weight patients with nuclear InsR $-/ \mathrm{ER}^{+}$tumors may thus benefit from less treatment than normal-weight patients with other nuclear InsR/ER combinations. Overweight or obese patients with nuclear InsR ${ }^{-/}$ $\mathrm{ER}^{-}$had the worst prognosis of all patients. Whether they may benefit from more tailored treatment, weight management, or better control of blood glucose levels remains to be elucidated.

\section{ETHICS STATEMENT}

This study was carried out in accordance with the recommendations of the local ethics committee at Lund University with written informed consent from all patients. All patients gave written informed consent in accordance with the Declaration of Helsinki. The protocol was approved by the local ethics committee at Lund University (Dnr 75-02, Dnr 37-08, Dnr 658-09, Dnr 58-12, Dnr 379-12, Dnr 227-13, Dnr 277-15, and Dnr 458-15).

\section{AUTHOR CONTRIBUTIONS}

Conception and design: SBj, AR, CR, CI, and HJ. Development of methodology and analysis and interpretation of data: $\mathrm{SBj}$,

\section{REFERENCES}

1. Jiralerspong S, Goodwin PJ. Obesity and breast cancer prognosis: evidence, challenges, and opportunities. JClin Oncol (2016) 34(35):4203-16. doi:10.1200/JCO.2016.68.4480

2. Patterson RE, Marinac CR, Natarajan L, Hartman SJ, Cadmus-Bertram L, Flatt SW, et al. Recruitment strategies, design, and participant characteristics in a trial of weight-loss and metformin in breast cancer survivors. Contemp Clin Trials (2016) 47:64-71. doi:10.1016/j.cct.2015.12.009

3. Engin A. Obesity-associated breast cancer: analysis of risk factors. Adv Exp Med Biol (2017) 960:571-606. doi:10.1007/978-3-319-48382-5_25

4. Guldberg TL, Christensen S, Zachariae R, Jensen AB. Prognostic factors in early breast cancer associated with body mass index, physical functioning, physical activity, and comorbidity: data from a nationwide Danish cohort. Breast Cancer Res Treat (2017) 162(1):159-67. doi:10.1007/s10549-016-4099-y
AR, and HJ. Acquisition of data: SBj, AR, MS, AM, CI, and HJ. Administrative, technical, or material support: SBj, AR, MS, AM, KJ, and HJ. Study supervision: CI and HJ. Writing, review, and/ or revision of the manuscript: $\mathrm{SBj}, \mathrm{AR}, \mathrm{MS}, \mathrm{AM}, \mathrm{KJ}, \mathrm{SB}, \mathrm{CR}, \mathrm{CI}$, and $\mathrm{HJ}$.

\section{ACKNOWLEDGMENTS}

The authors thank research nurses Anette Ahlin Gullers, Monika Meszaros, Maj-Britt Hedenblad, Karin Henriksson, Anette Möller, Helén Thell, Jessica Åkesson, and Linda Ågren. They also thank Erika Bågeman, Maria Henningson, and Maria Hjertberg for data entry, Björn Nodin and Elise Nilsson for TMA construction, Kristina Lövgren for staining, and Catarina Blennow for sectioning, as well as breast pathologist Anna Ehinger for help with histopathological assessments.

\section{FUNDING}

This work was supported by grants from The Swedish Cancer Society (CAN2014/465) (Helena Jernström); the Mrs. Berta Kamprad Foundation (BKS19/2014, BKS27/2015) (Helena Jernström); the Gunnar Nilsson Foundation (Helena Jernström); the Swedish Breast Cancer Group (BRO) (Christian Ingvar); the South Swedish Health Care Region (Region Skåne ALF 10622) (Helena Jernström); Konung Gustaf V:s Jubileumsfond (Helena Jernström); the Lund Hospital Fund (Christian Ingvar and Helena Jernström), the RATHER consortium (http://www.ratherproject.com/), and the Seventh Framework program (William. Gallagher@ucd.ie).The funding agencies played no role in design of the study; the collection, analysis, and interpretation of the data; the writing of the manuscript; or the decision to submit the manuscript for publication.

\section{SUPPLEMENTARY MATERIAL}

The Supplementary Material for this article can be found online at http://www.frontiersin.org/article/10.3389/fendo.2017.00332/ full\#supplementary-material.

FIGURE S1 | Representative images of cytoplasmic, membrane and nuclear InsR staining in breast cancer tissue, scale bar $=20 \mu \mathrm{m}$.

5. Park J, Morley TS, Kim M, Clegg DJ, Scherer PE. Obesity and cancer mechanisms underlying tumour progression and recurrence. Nat Rev Endocrinol (2014) 10(8):455-65. doi:10.1038/nrendo.2014.94

6. Gletsu NA, Field CJ, Clandinin MT. Obese mice have higher insulin receptor levels in the hepatocyte cell nucleus following insulin stimulation in vivo with an oral glucose meal. Biochim Biophys Acta (1999) 1454(3):251-60. doi:10.1016/S0925-4439(99)00043-5

7. Pandini G, Vigneri R, Costantino A, Frasca F, Ippolito A, Fujita-Yamaguchi Y, et al. Insulin and insulin-like growth factor-I (IGF-I) receptor overexpression in breast cancers leads to insulin/IGF-I hybrid receptor overexpression: evidence for a second mechanism of IGF-I signaling. Clin Cancer Res (1999) 5(7):1935-44.

8. Pollak M. The insulin and insulin-like growth factor receptor family in neoplasia: an update. Nat Rev Cancer (2012) 12(3):159-69. doi:10.1038/ $\operatorname{nrc} 3215$ 
9. Björner S, Rosendahl AH, Simonsson M, Markkula A, Jirström K, Borgquist S, et al. Combined and individual tumor-specific expression of insulin-like growth factor-I receptor, insulin receptor and phospho-insulin-like growth factor-I receptor/insulin receptor in primary breast cancer: implications for prognosis in different treatment groups. Oncotarget (2017) 8(6):9093-107. doi:10.18632/oncotarget.14082

10. Law JH, Habibi G, Hu K, Masoudi H, Wang MY, Stratford AL, et al. Phosphorylated insulin-like growth factor-i/insulin receptor is present in all breast cancer subtypes and is related to poor survival. Cancer Res (2008) 68(24):10238-46. doi:10.1158/0008-5472.CAN-08-2755

11. Gradishar WJ, Yardley DA, Layman R, Sparano JA, Chuang E, Northfelt DW, et al. Clinical and translational results of a phase II, randomized trial of an anti-IGF-1R (cixutumumab) in women with breast cancer that progressed on endocrine therapy. Clin Cancer Res (2016) 22(2):301-9. doi:10.1158/ 1078-0432.CCR-15-0588

12. Wing SS. The UPS in diabetes and obesity. BMC Biochem (2008) 9(Suppl 1):S6. doi:10.1186/1471-2091-9-S1-S6

13. Haeusler RA, McGraw TE, Accili D. Biochemical and cellular properties of insulin receptor signalling. Nat Rev Mol Cell Biol (2017). doi:10.1038/ nrm.2017.89

14. Podlecki DA, Smith RM, Kao M, Tsai P, Huecksteadt T, Brandenburg D, et al. Nuclear translocation of the insulin receptor. A possible mediator of insulin's long term effects. J Biol Chem (1987) 262(7):3362-8.

15. Nelson JD, LeBoeuf RC, Bomsztyk K. Direct recruitment of insulin receptor and ERK signaling cascade to insulin-inducible gene loci. Diabetes (2011) 60(1):127-37. doi:10.2337/db09-1806

16. Sarfstein R, Pasmanik-Chor M, Yeheskel A, Edry L, Shomron N, Warman N, et al. Insulin-like growth factor-I receptor (IGF-IR) translocates to nucleus and autoregulates IGF-IR gene expression in breast cancer cells. J Biol Chem (2012) 287(4):2766-76. doi:10.1074/jbc.M111.281782

17. Solomon-Zemler R, Sarfstein R, Werner H. Nuclear insulin-like growth factor-1 receptor (IGF1R) displays proliferative and regulatory activities in non-malignant cells. PLoS One (2017) 12(9):e0185164. doi:10.1371/journal. pone. 0185164

18. Kahlert S, Nuedling S, van Eickels M, Vetter H, Meyer R, Grohe C. Estrogen receptor alpha rapidly activates the IGF-1 receptor pathway. J Biol Chem (2000) 275(24):18447-53. doi:10.1074/jbc.M910345199

19. Stoica A, Saceda M, Fakhro A, Joyner M, Martin MB. Role of insulin-like growth factor-I in regulating estrogen receptor-alpha gene expression. J Cell Biochem (2000) 76(4):605-14. doi:10.1002/(SICI)1097-4644(20000315)76: 4<605::AID-JCB9>3.0.CO;2-T

20. Yee D, Lee AV. Crosstalk between the insulin-like growth factors and estrogens in breast cancer. J Mammary Gland Biol Neoplasia (2000) 5(1):107-15. doi:10.1023/A:1009575518338

21. Enger SM, Ross RK, Paganini-Hill A, Carpenter CL, Bernstein L. Body size, physical activity, and breast cancer hormone receptor status: results from two case-control studies. Cancer Epidemiol Biomarkers Prev (2000) 9(7):681-7.

22. Yang XR, Chang-Claude J, Goode EL, Couch FJ, Nevanlinna H, Milne RL, et al. Associations of breast cancer risk factors with tumor subtypes: a pooled analysis from the Breast Cancer Association Consortium studies. J Natl Cancer Inst (2011) 103(3):250-63. doi:10.1093/jnci/djq526

23. Kakugawa Y, Tada H, Kawai M, Suzuki T, Nishino Y, Kanemura S, et al. Associations of obesity and physical activity with serum and intratumoral sex steroid hormone levels among postmenopausal women with breast cancer: analysis of paired serum and tumor tissue samples. Breast Cancer Res Treat (2017) 162(1):115-25. doi:10.1007/s10549-016-4094-3

24. Jernström $H$, Olsson $H$. Insulin-like growth factor-1 in relation to adult weight and birth weight in healthy nulliparous women. Int $J$ Gynaecol Obstet (1998) 62(1):11-8.

25. Jernström H, Barrett-Connor E. Obesity, weight change, fasting insulin, proinsulin, C-peptide, and insulin-like growth factor-1 levels in women with and without breast cancer: the Rancho Bernardo Study. J Womens Health Gend Based Med (1999) 8(10):1265-72. doi:10.1089/jwh.1.1999.8.1265

26. Carpentier JL, Fehlmann M, Van Obberghen E, Gorden P, Orci L. Insulin receptor internalization and recycling: mechanism and significance. Biochimie (1985) 67(10-11):1143-5. doi:10.1016/S0300-9084(85)80112-7

27. Kublaoui B, Lee J, Pilch PF. Dynamics of signaling during insulinstimulated endocytosis of its receptor in adipocytes. J Biol Chem (1995) 270(1):59-65. doi:10.1074/jbc.270.1.59
28. Yee D. A tale of two receptors: insulin and insulin-like growth factor signaling in cancer. Clin Cancer Res (2015) 21(4):667-9. doi:10.1158/1078-0432.CCR14-2056

29. Simonsson M, Söderlind V, Henningson M, Hjertberg M, Rose C, Ingvar C, et al. Coffee prevents early events in tamoxifen-treated breast cancer patients and modulates hormone receptor status. Cancer Causes Control (2013) 24(5):929-40. doi:10.1007/s10552-013-0169-1

30. Ringberg A, Bågeman E, Rose C, Ingvar C, Jernström H. Of cup and bra size: reply to a prospective study of breast size and premenopausal breast cancer incidence. Int J Cancer (2006) 119(9):2242-43; author reply 2244. doi:10.1002/ ijc. 22104

31. Markkula A, Bromee A, Henningson M, Hietala M, Ringberg A, Ingvar C, et al. Given breast cancer, does breast size matter? Data from a prospective breast cancer cohort. Cancer Causes Control (2012) 23(8):1307-16. doi:10.1007/s10552-012-0008-9

32. Bågeman E, Ingvar C, Rose C, Jernström H. Coffee consumption and CYP1A2 1 F genotype modify age at breast cancer diagnosis and estrogen receptor status. Cancer Epidemiol Biomarkers Prev (2008) 17(4):895-901. doi:10.1158/1055-9965.EPI-07-0555

33. Jernström H, Bågeman E, Rose C, Jönsson PE, Ingvar C. CYP2C8 and CYP2C9 polymorphisms in relation to tumour characteristics and early breast cancer related events among 652 breast cancer patients. Br J Cancer (2009) 101(11):1817-23. doi:10.1038/sj.bjc.6605428

34. Simonsson M, Markkula A, Bendahl PO, Rose C, Ingvar C, Jernström H. Pre- and postoperative alcohol consumption in breast cancer patients: impact on early events. Springerplus (2014) 3:261. doi:10.1186/2193-1801-3-261

35. McShane LM, Altman DG, Sauerbrei W, Taube SE, Gion M, Clark GM, et al. REporting recommendations for tumor MARKer prognostic studies (REMARK). Nat Clin Pract Urol (2005) 2(8):416-22. doi:10.1038/ ncponc0252

36. Dupont WD, Plummer WD Jr. Power and sample size calculations for studies involving linear regression. Control Clin Trials (1998) 19(6):589-601. doi:10.1016/S0197-2456(98)00037-3

37. Hawsawi Y, El-Gendy R, Twelves C, Speirs V, Beattie J. Insulin-like growth factor - oestradiol crosstalk and mammary gland tumourigenesis. Biochim Biophys Acta (2013) 1836(2):345-53. doi:10.1016/j.bbcan.2013.10.005

38. Crudden C, Girnita A, Girnita L. Targeting the IGF-1R: the tale of the tortoise and the hare. Front Endocrinol (2015) 6:64. doi:10.3389/fendo. 2015.00064

39. Hair BY, Xu Z, Kirk EL, Harlid S, Sandhu R, Robinson WR, et al. Body mass index associated with genome-wide methylation in breast tissue. Breast Cancer Res Treat (2015) 151(2):453-63. doi:10.1007/s10549-015-3401-8

40. Dabrosin C. Increase of free insulin-like growth factor-1 in normal human breast in vivo late in the menstrual cycle. Breast Cancer Res Treat (2003) 80(2):193-8. doi:10.1023/A:1024575103524

41. Bertacca A, Ciccarone A, Cecchetti P, Vianello B, Laurenza I, Del Prato S, et al. High insulin levels impair intracellular receptor trafficking in human cultured myoblasts. Diabetes Res Clin Pract (2007) 78(3):316-23. doi:10.1016/ j.diabres.2007.06.001

42. Sorlie T, Perou CM, Tibshirani R, Aas T, Geisler S, Johnsen H, et al. Gene expression patterns of breast carcinomas distinguish tumor subclasses with clinical implications. Proc Natl Acad Sci U S A (2001) 98(19):10869-74. doi:10.1073/pnas.191367098

43. Agrawal S, Luc M, Ziolkowski P, Agrawal AK, Pielka E, Walaszek K, et al. Insulin-induced enhancement of MCF-7 breast cancer cell response to 5-fluorouracil and cyclophosphamide. Tumour Biol (2017) 39(6): 1010428317702901. doi:10.1177/1010428317702901

44. Fox EM, Miller TW, Balko JM, Kuba MG, Sanchez V, Smith RA, et al. A kinome-wide screen identifies the insulin/IGF-I receptor pathway as a mechanism of escape from hormone dependence in breast cancer. Cancer Res (2011) 71(21):6773-84. doi:10.1158/0008-5472.CAN-11-1295

45. Olanders KJ, Lundgren GA, Johansson AM. Betamethasone in prevention of postoperative nausea and vomiting following breast surgery. J Clin Anesth (2014) 26(6):461-5. doi:10.1016/j.jclinane.2014.02.006

46. Jolley JA, Rajan PV, Petersen R, Fong A, Wing DA. Effect of antenatal betamethasone on blood glucose levels in women with and without diabetes. Diabetes Res Clin Pract (2016) 118:98-104. doi:10.1016/j.diabres.2016.06.005

47. Lundin KB, Henningson M, Hietala M, Ingvar C, Rose C, Jernström H. Androgen receptor genotypes predict response to endocrine treatment in 
breast cancer patients. Br J Cancer (2011) 105(11):1676-83. doi:10.1038/ bjc.2011.441

48. Persson M, Simonsson M, Markkula A, Rose C, Ingvar C, Jernström H. Impacts of smoking on endocrine treatment response in a prospective breast cancer cohort. Br J Cancer (2016) 115(3):382-90. doi:10.1038/bjc. 2016.174

49. Griggs JJ, Mangu PB, Anderson H, Balaban EP, Dignam JJ, Hryniuk WM, et al. Appropriate chemotherapy dosing for obese adult patients with cancer: American Society of Clinical Oncology clinical practice guideline. JClin Oncol (2012) 30(13):1553-61. doi:10.1200/JCO.2011.39.9436
Conflict of Interest Statement: SB has consultant fee honoraria from Novartis and Roche.

Copyright ๑ 2017 Björner, Rosendahl, Simonsson, Markkula, Jirström, Borgquist, Rose, Ingvar and Jernström. This is an open-access article distributed under the terms of the Creative Commons Attribution License (CC BY). The use, distribution or reproduction in other forums is permitted, provided the original author(s) or licensor are credited and that the original publication in this journal is cited, in accordance with accepted academic practice. No use, distribution or reproduction is permitted which does not comply with these terms. 